\title{
The Calibration of the Geometry and Antenna delay in Askaryan Radio Array Station 4 and 5
}

\author{
Paramita Dasgupta ${ }^{a, *}$ Kaeli Hughes ${ }^{b}$ on behalf of the ARA Collaboration \\ (a complete list of authors can be found at the end of the proceedings) \\ ${ }^{a}$ Université Libre de Bruxelles, Science Faculty CP230, B-1050 Brussels, Belgium \\ ${ }^{b}$ Dept. of Physics, Enrico Fermi Inst., Kavli Inst. for Cosmological Physics, University of Chicago, \\ Chicago, IL 60637, U.S.A. \\ E-mail: paramita.dasgupta@ulb.be, kahuges@uchicago.edu
}

The Askaryan Radio Array (ARA) experiment at the South Pole is designed to detect the radio signals produced by ultra high energy cosmic neutrino interactions in the ice. There are 5 independent ARA stations, one of which (A5) includes a low-threshold phased array trigger string. Each ARA station is designed to work as an autonomous detector. The Data Acquisition System in all ARA stations is equipped with the Ice Ray Sampler second-generation (IRS2) chip, a custom-made, application-specific integrated circuit (ASIC) for high-speed sampling and digitization. In this contribution, we describe the methodology used to calibrate the IRS 2 digitizer chip and the station geometry, namely the relative timing between each pair of ARA antennas, deployed at $200 \mathrm{~m}$ below the Antarctic ice surface, and their geometrical positions in the ice, for ARA stations 4 and 5. Our calibration allows for proper timing correlations between incoming signals, which is crucial for radio vertex reconstruction and thus detection of ultra high energy neutrinos through the Askaryan effect. We achieve a signal timing precision on a sub-nanosecond level and an antenna position precision within $10 \mathrm{~cm}$.

37th International Cosmic Ray Conference (ICRC 2021)

July 12th - 23rd, 2021

Online - Berlin, Germany

\footnotetext{
${ }^{*}$ Presenter
} 


\section{The ARA Instrument}

The study of ultra high energy (UHE) neutrinos (neutrino energy, $E_{v}>10^{17} \mathrm{eV}$ ) is intriguing as they carry exclusive information about the ultra high energy cosmic rays. Several ongoing experiments such as the IceCube [1], ARA [2], ANITA [3], ARIANNA [4], and also the RNOG [5], currently under construction at Greenland, aim to detect ultra high energy neutrinos.

The Askaryan Radio Array is designed to detect in-ice UHE neutrino-induced particle showers through the Askaryan effect [6]. ARA is located a few kilometers grid-west of the geographic South Pole in Antarctica, next to the IceCube experiment. Currently, there are 5 ARA stations (A1-A5) deployed with a spacing of $2 \mathrm{~km}$ between them, to maximize the effective area at $E_{v}>10^{18} \mathrm{eV}$.
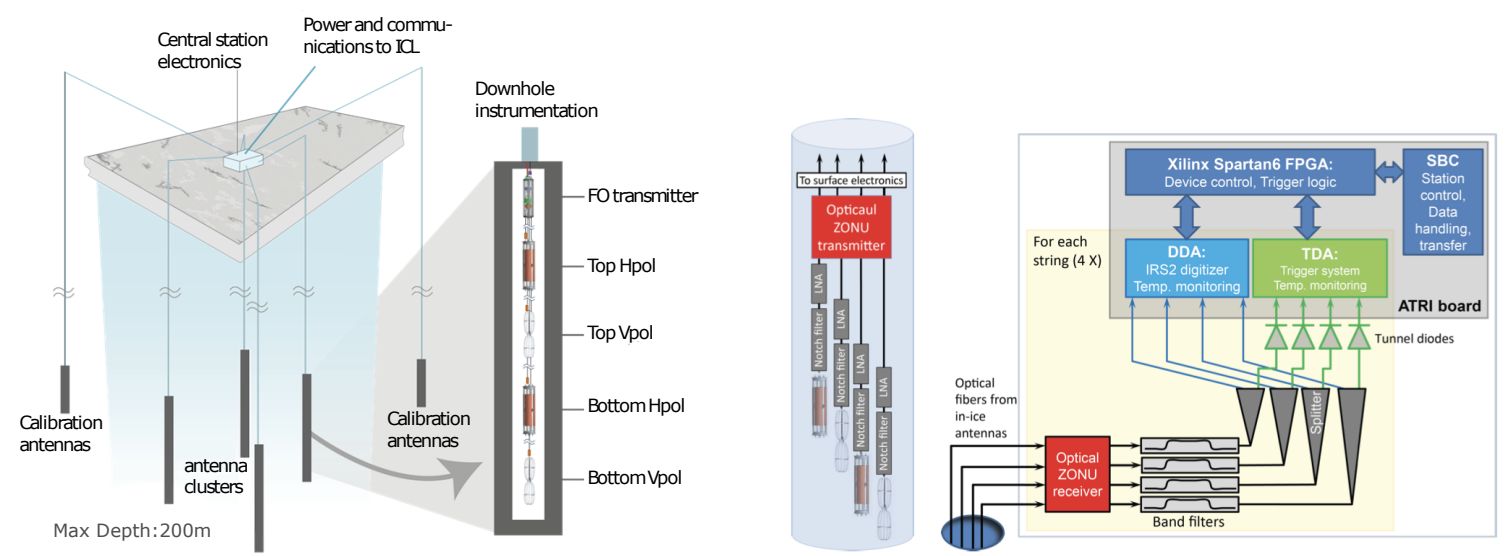

Figure 1: Left: A schematic of the electronics and instrumentation in an ARA station; "FO" stands for the fiber-optic transmitter. Right: The components of the down-hole signal chain on each string in the ARA stations and the surface Data acquisition system of the ARA stations. Images are taken from [2].

Each ARA station comprises 16 antennas, deployed at the bottom of four $200 \mathrm{~m}$ deep, vertical holes on 4 "measurement strings", with each string comprising of 2 VPol, and 2 HPol antennas (Fig. 1, left). The VPol and HPol antennas, capable of recording data in the range $150 \mathrm{MHz}-$ $850 \mathrm{MHz}$, are used to detect vertically-polarized and horizontally-polarized radio frequency (RF) signals respectively [2].

In addition to the 16 receiver antennas, each ARA station is equipped with 2 extra strings, known as the "calibration strings", deployed at the same depth as the 4 measurement strings, and located at a distance of about $40 \mathrm{~m}$ from the core of the station (Fig. 1, left). Each calibration string contains two transmitter antennas, one HPol, and one VPol both emitting broadband RF pulses, or continuous RF noise for in-situ calibration of station geometry and timing.

\section{The ARA Digitization System}

The RF signals, recorded by the ARA antennas in the ice, are first filtered by a bandpass and notch filter to remove South Pole Station communications [7]. The filtered signals are then amplified and transmitted to the surface through RF over Fiber that provides minimal signal attenuation (Fig. 1, right). At the surface, signals are again filtered and amplified before they are split into two paths, 
to the triggering system which is mounted on the Triggering Daughter board for ARA (TDA), and to the digitization system which is mounted on the Digitizing Daughter board for ARA (DDA) (Fig. 1, right). Both the TDA and DDA boards are mounted on the ARA Triggering and Readout Interface (ATRI) which provides all logic for the data acquisition systems in a single Spartan-6 FPGA (Fig. 1, right). The ARA triggering system determines whether a signal should be recorded, based on whether the signal is registered on 3 out of 8 antennas of the same polarization. A signal that passes a triggering condition is recorded in the digitization path (Fig. 1, right).

\section{Calibration of ARA Station 4 and 5 Digitizers}

The input RF signal is sampled at a speed of $\sim 3.2 \mathrm{GS} / \mathrm{s}$ by the Ice Ray Sampler secondgeneration (IRS2) digitizer chip. IRS2 is a custom-made, application-specific integrated circuit (ASIC), providing high-speed digitization at low power consumption $(\sim 20 \mathrm{~mW}$ per channel). The ARA digitization system consists of 4 IRS2 digitizer chips (DDA0-DDA3) with 4 channels per chip to sample the input data. Prior to the data analysis, proper calibration of all 16 channels of the IRS2 digitizer chips is essential.

The IRS2 chip stores the input signal via the Switched Capacitor Array (SCA), consisting of 128 sampling capacitors per channel, equally divided into 64 even and 64 odd samples on two delay lines [8]. The SCA utilizes finely tuned 128 delay elements per channel to set up a sampling sequence of the input data. However, these delay elements can have offsets of several $100 \mathrm{ps}$ due to the variation in the chip fabrication [7]. These offsets can disturb the timing of the sampled input waveform, thus, adding high frequency noise components and making the waveforms less precise for further analysis. Hence, we need to correct these timing offsets (or "jitter") for individual 128 sampling capacitors per channel of the digitizer chip.

\subsection{Timing Calibration}

For both timing and amplitude calibration (Sec. 3.2), pre-deployment sine waves of several amplitudes and frequencies $(200 \mathrm{MHz}-800 \mathrm{MHz})$ have been generated in runs of about 4000 waveforms with an external waveform generator and fed to the IRS2 chip, mounted on a DDA. The instrument has been kept at $-50^{\circ} \mathrm{C}$ to emulate the operational conditions of polar ice. We use these calibration data sets to correct the individual timing offset of 128 sampling capacitors per channel in the A4 and A5 stations. We briefly describe the timing calibration process below.

1. Each waveform in a given calibration data run is fitted to a sine wave and the fit frequency is compared to the known input frequency $\left(f_{I}\right)$ of the calibration data, chosen at $218 \mathrm{MHz}$ for this work. We histogram the fit frequencies, obtained by fitting all waveforms in a calibration run to sine waves of input frequency $f_{I}$. Using the average fit frequency $\left(f_{a v}\right)$, we scale the average time step between samples by the ratio $\left(f_{a v} / f_{I}\right)$.

2. We determine the timing offset or jitters which is the difference between the recorded time and the expected time, for each of the 128 sampling capacitors and correct the input event time array by adding the timing offset for each sample to the original time step of that sample. We iterate this correction several times until the correction factor converges. 
In the 1st iteration, we find that the timing offset for each even sample (Fig. 2a), and each odd sample (Fig. 2c), is widely spread around the center at $0 \mathrm{~ns}$. This spread is expected, since the timing offset is only determined in this step, and not yet corrected.

Typically, after 5-6 iterations, we obtain a stable precision in timing for all 4 channels of each DDA in both A4 and A5 stations. The final jitter histogram for all 64 even samples and all 64 odd samples in channel "0", DDA0 of ARA station 4 is shown in Fig. 2b and Fig. 2d respectively. We find that the final timing jitter is centered at $0 \mathrm{~ns}$ for all 128 sampling elements, expected from a proper timing calibration. We obtain similar results for the A5 station.

The observed spread and bimodal behavior (Fig. 2b), present prominently in the even samples is related to a non-linearity in the voltage (Sec. 3.2) of the respective sample [8]. We also observe that, this non-linearity and asymmetry increase with the increase in channel number. The performance of the chip worsens as the channel number increases, as not only the even samples but also the odd samples show bimodal behavior in higher channels of the chip, in particular in channel " 3 " of all IRS2 digitizer chips in both A4 and A5 stations.

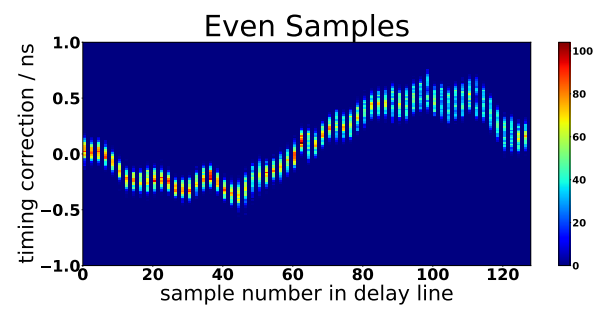

(a)

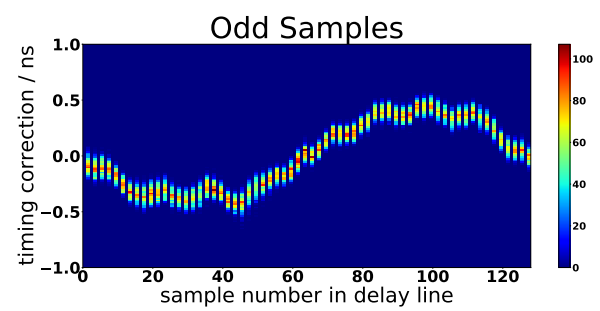

(c)

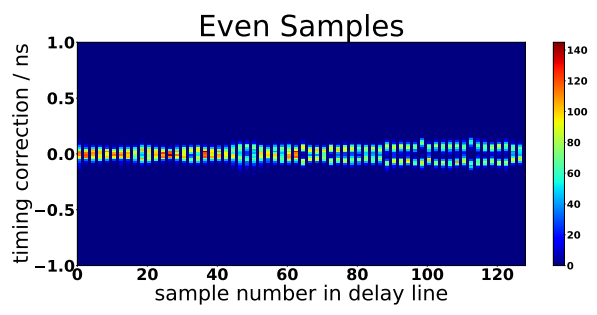

(b)

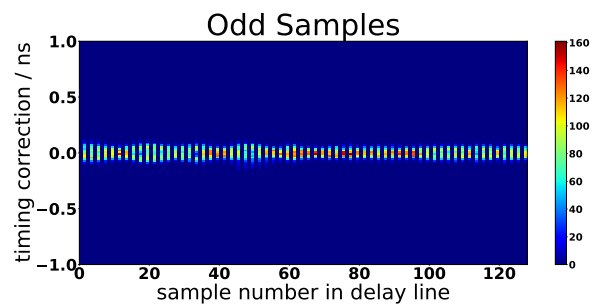

(d)

Figure 2: Top: The timing corrections for 64 even samples in BVPol (channel "0", DDA0) in the A4 station, a) before timing calibration is performed, and b) after timing calibration is performed. Bottom: The timing corrections for 64 odd samples of the same channel in the A4 station as in the top panel, c) before timing calibration is performed, and d) after timing calibration is performed.

\subsection{Voltage Calibration}

In addition to the timing corrections, the ADC-to-voltage conversion factor needs to be determined for all 32-k storage capacitors in each channel of the IRS2 digitizer chips. Each channel of the IRS2 digitizer chip has 32,768 storage cells which are further subdivided into 512 blocks. Hence, each of these 512 blocks has 64 storage cells that store the input RF data. For voltage calibration, the timing-corrected calibration data are used as input. We fit each timing-corrected waveform from a chosen pre-deployment calibration run of known input amplitude to a sine wave, 
keeping the frequency and the phase of the waveforms as free parameters, and predict the input voltage for each $\mathrm{ADC}$ point. In the next step, we histogram all ADC points and the corresponding input voltage points for each 32-k storage sample to have a statistically significant collection of data points per storage cell to find the ADC-to-voltage conversion factor.

For each A5 digitizer channel, the collected data are first separated into bins of $30 \mathrm{mV}$, and the average data point per bin is obtained. The averaged points are then fitted with a broken polynomial fit of order 3 (Fig. 3, left). This method is applied to all 32-k storage cells per channels of the A5 digitizer. The same fitting procedure has been previously implemented in the case of two ARA stations (A2 and A3) to account for the non-linearity and bimodal behavior observed in some samples of the chip [8].
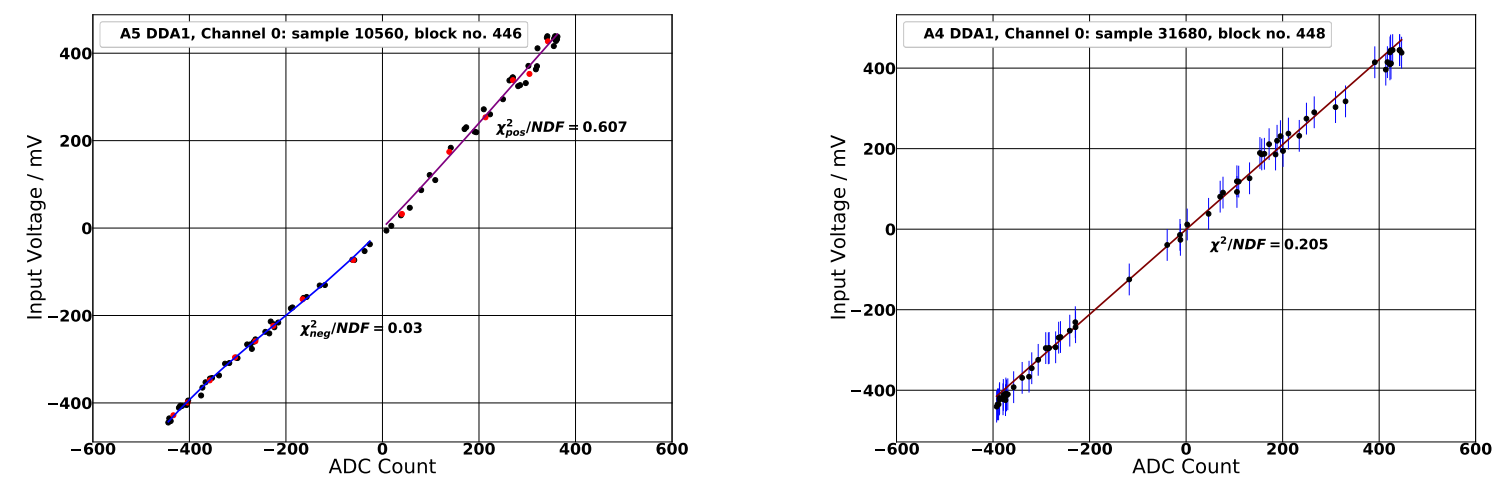

Figure 3: Left: Collected data (black points), averaged data (red points), and a broken 3rd order polynomial fit. The purple line shows the positive half of the broken fit, and the blue line shows the negative half of the broken fit, for one storage sample in a BVPol channel (channel "0", DDA1) in the A5 station. Right: Calibration data (black points) with errors (blue lines) and the linear fit (red line) to the data points, for one storage sample in a BVPol channel (channel "0", DDA1) in the A4 station.

For A4 digitizer channels, we find that a linear fit between ADC and voltage points without averaging them can render results similar to the results obtained with the polynomial fit in case of the A5 digitizer channels. This linear fitting method involves two iterations. In the first iteration, we fit the ADC to the input voltage points and find the fit parameters as well as the residual for each input voltage point per sample. In the next iteration, we re-fit the ADC and the input voltage points per sample with a linear fit and the error, estimated from the maximum residual for that sample. This method is applied to individual storage cell to find the ADC-to-voltage conversion factor for all 32,768 storage cells in each A4 digitizer chip (Fig. 3, right).

We determine two separate $\chi^{2} / N D F$, i.e, $\chi_{\text {pos }}^{2} / N D F$ and $\chi_{\text {neg }}^{2} / N D F$ for the positive and the negative half of the broken fit respectively for each storage sample in A5 digitizers (Fig. 3, left) and the $\chi^{2} / N D F$ for each storage sample in A4 digitizers (Fig. 3, right). It is observed that a randomly chosen storage sample from any of the 512 blocks normally behaves in a very similar manner as the sample at the same position in the neighboring blocks [8]. Therefore, the fit parameters for samples with the $\chi^{2} / N D F>1$ are discarded and the ADC-to-voltage conversion factor of the sample with the same sample number in a neighboring block is taken, provided it has a $\chi^{2} / N D F$ value $\leq 1$. In this way, we achieve a relatively stable voltage calibration in all channels of the A4 and A5 digitizers. 


\subsection{Cross-check Calibration with Calibration Pulser Waveforms}

To validate our calibration methods, we use physics signals from the calibration antennas (Sec. 1) and perform two checks. First, we apply i) only timing corrections (Sec. 3.1), and ii) both timing and voltage corrections (Sec. 3.2) on the input signals from the calibration antenna. We compare the uncalibrated and the calibrated waveforms in each channels of the digitizers to investigate the effects of calibration on the input RF signals (Fig. 4).

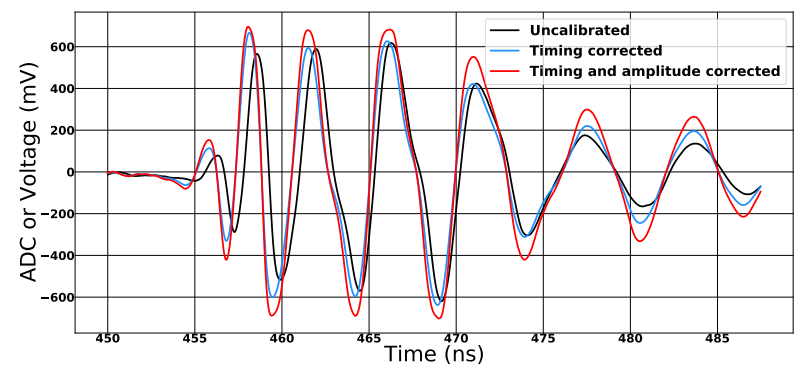

Figure 4: Cross-check digitizer calibration with the uncalibrated (black), only timing corrected (blue), and fully calibrated RF signals (red)

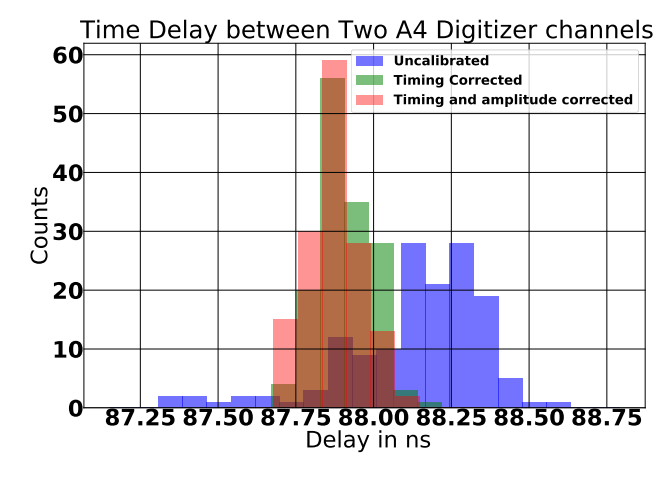

(a)

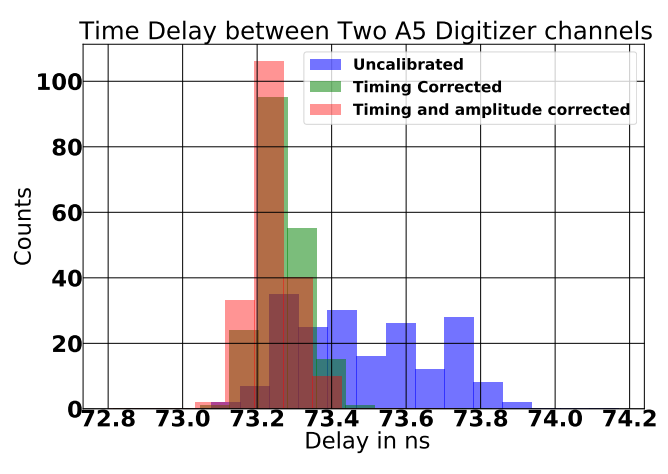

(b)

Figure 5: The time delay between two VPol channels in (a) an A4 digitizer chip and (b) an A5 digitizer chip.

Next, we find the inter-channel time delay for all pairs of channels in A4 and A5 digitizers using i) uncalibrated signal, ii) only timing calibrated signal, and iii) both timing and amplitude calibrated signals. In Figs. 5a and 5b, we show the significant improvement in the precision of the inter-channel time delay with the use of fully calibrated RF signals as input, for A4 and A5 stations respectively. These results validate our calibration methodology that provides precise timing correlations between incoming signals.

\section{Calibration of the ARA Station 5 Geometry}

The final step in calibrating an ARA station is to determine the antenna positions within the ice. Antenna positions must be known to within $5 \mathrm{~cm}$ so that the signals coherently sum across the 


\begin{tabular}{|c|c|c|}
\hline Uncertainty Type & Estimated Error & How to Improve \\
\hline Ice Model & $\begin{array}{l}+/-0.05 \mathrm{~ns} ; \text { additional } \\
\text { systematic offset larger than } \\
\text { statistical error }\end{array}$ & $\begin{array}{l}\text { More precise measurements } \\
\text { of cable lengths and cables } \\
\text { speeds at cold temperatures } \\
\text { prior to deployment }\end{array}$ \\
\hline $\begin{array}{l}\text { Traditional ARA } 5 \\
\text { Calibration Pulser time } \\
\text { delays }\end{array}$ & $+/-0.082 \mathrm{~ns}$ & $\begin{array}{l}\text { More identical antenna } \\
\text { impedances; better in situ } \\
\text { measurements; improved } \\
\text { digitizers }\end{array}$ \\
\hline $\begin{array}{ll}\text { Phased } & \text { Array } \\
\text { Calibration } & \text { Pulser } \\
\text { time delays } & \end{array}$ & $+/-0.03 \mathrm{~ns}$ & None \\
\hline Minuit Fit & $<5 \mathrm{~cm}$ & $\begin{array}{l}\text { Orthogonal } \\
\text { pulsers to find minimum } \\
\text { faster }\end{array}$ \\
\hline
\end{tabular}

Table 1: A summary of the precision obtained by each step of the ARA Station 5 Position Calibration

entire frequency band, which means that positions, usually surveyed to the meter, must be corrected using calibration sources. Additionally, the ARA Station 5 has the added problem of needing relative positions between every antenna, including both the traditional ARA strings as well as the separate phased array string. This process involves simultaneously fitting the cable delays, antenna positions, and ice model, which each carry their own approximate uncertainties. At present, only ARA Station 5 has had its antenna positions fully calibrated; A4 is a work in progress.

There are four main sources of error in calibrating the station positions, laid out in Table 1. The first is the ice model, which has typically been modeled using a depth-dependent exponential model. There have been many attempts to measure the index of refraction of the South Pole ice; to determine which measurement most accurately reflected the ice near ARA Station 5, the vertical distance between the phased array antennas was assumed to be known exactly. This is a reasonable assumption because each antenna is deployed with only 1-meter spacing, and each position is recorded with centimeter precision. Using the phased array and a calibration source at the SPIceCore hole about $4 \mathrm{~km}$ away, the predicted time delays from many ice models were compared against the time delays measured by the phased array. The model that fit the data best was an internal ARA model developed in 2016. One parameter of this model has been modified further to obtain the best fitting model possible. At this point, the exact distance between the local calibration pulser string and the phased array string has been also optimized and found to be $52 \mathrm{~m}$.

The next source of error is the precision of the time delays for both the traditional ARA channels as well as the phased array channels. The average error for an ARA station 5 channel pair is 82 ps; the average error for an ARA Station 4 channel pair is 90 ps. Both of these are less precise than that of the phased array, which was deployed with a more modern digitizer and more identical impedances on each channel, and obtained a 30 ps Gaussian spread around each of its calculated 
time delays. Finally, two separate Minuit fits have been used to calculate the relative location of the deployed strings. ARA Station 5 is limited to only one local calibration pulser, which is not enough to uniquely constrain the positions of the antennas. Instead, data from a SPIceCore calibration drop taken in December 2018 is used as a secondary calibration source. The SPIceCore hole is also used as the reference location, as its position is well-recorded and known with respect to other landmarks such as the IceCube Lab building and the South Pole Station. Because the traditional ARA station and the phased array trigger are technically two separate systems, the fit needs to be done in two rounds: one with only the ARA channels, and another to fit the relative location of the ARA 5 strings with respect to the phased array. This relative timing is possible because one ARA channel was split and sent into both A5 and the phased array, and can be used to line up signals seen by both systems.

Using Minuit, each of the $(x, y, z)$ coordinates of the antennas are found within $5 \mathrm{~cm}$, based on Hessian error functionality built into the Minuit program. This meets the requirements for antenna position calibration, as a $5 \mathrm{~cm}$ window is small enough to allow signals with frequencies up to 1000 $\mathrm{MHz}$ to sum coherently.

\section{Summary and Outlook}

In this proceeding, we describe our calibration methodology for the ARA Digitization system. We describe the calibration of the ARA station geometry and show the results for antenna delay calculation for ARA station 5. Our calibration technique allows us to achieve proper timing correlations between incoming signals, which is crucial for radio vertex reconstruction and thus detection of ultra high energy neutrinos. We show that a signal timing precision of $\sim 100 \mathrm{ps}$ and an antenna position precision within $10 \mathrm{~cm}$ are achieved through our calibration methods.

\section{References}

[1] IceCube 2 Collaboration, M. G. Aartsen et al. Phys. Rev. D 98 (Sep, 2018) 062003.

[2] ARA Collaboration, P. Allison et al. Phys. Rev. D 102 (2020) 043021.

[3] ANITA Collaboration, S. Prohira, A. Novikov, P. Dasgupta, P. Jain, et al. Phys. Rev. D 98 (Aug, 2018) 042004.

[4] A. Anker et al. JCAP 03 (2020) 053.

[5] RNO-G Collaboration, J. A. Aguilar, P. Allison, J. J. Beatty, H. Bernhoff, D. Besson, et al. Journal of Instrumentation 16 no. 3, (Mar., 2021) P03025.

[6] G. A. Askar'yan Zh. Eksp. Teor. Fiz. 41 (1961) 616-618.

[7] ARA Collaboration, P. Allison et al. Phys. Rev. D 93 (2016) 082003.

[8] T. Meures, Development of a Sub-glacial Radio Telescope for the Detection of GZK Neutrinos. Springer International Publishing, 01, 2015. 


\section{Full Author List: ARA Collaboration}

P. Allison ${ }^{15}$, S. Archambault ${ }^{17}$, J.J. Beatty ${ }^{15}$, M. Beheler-Amass ${ }^{4}$, D.Z. Besson ${ }^{10,13}$, M. Beydler ${ }^{4}$, C.H. Chen ${ }^{2}$, P. Chen ${ }^{2}$, Y.C. Chen ${ }^{2}$, B.A. Clark ${ }^{16}$, W. Clay $^{8}$, A. Connolly ${ }^{15}$, L. Cremonesi ${ }^{6}$, P. Dasgupta ${ }^{18}$, J. Davies ${ }^{6}$, S. De Kockere ${ }^{9}$, K.D. de Vries ${ }^{9}$, C. Deaconu ${ }^{8}$, M. A. DuVernois ${ }^{4}$, J. Flaherty ${ }^{15}$, E. Friedman ${ }^{3}$, R. Gaior ${ }^{17}$, J. C. Hanson ${ }^{19}$, K. Hanson ${ }^{4}$, N. Harty ${ }^{1}$, B. Hendricks ${ }^{11,12}$, K.D. Hoffman ${ }^{3}$, B. Hokanson-Fasig ${ }^{4}$, E. Hong ${ }^{15}$, S.Y. Hsu ${ }^{2}$, J.J. Huang ${ }^{2}$, M.-H. Huang ${ }^{2}$, K. Hughes ${ }^{8}$, A. Ishihara ${ }^{17}$, A. Karle ${ }^{4}$, J.L. Kelley ${ }^{4}$, R. Khandelwal ${ }^{4}$, K.-C. Kim $^{3}$, M.-C. Kim ${ }^{17}$, R. Krebs ${ }^{11,12}$, I. Kravchenko ${ }^{14}$, Y. Ku ${ }^{11,12}$, C.Y. Kuo ${ }^{2}$, K. Kurusu ${ }^{17}$, U.A. Latif ${ }^{10,9}$, A. Laundrie ${ }^{4}$, H. Landsman ${ }^{7}$, M.-Y. Lu ${ }^{4}$, T.-C. Liu ${ }^{2}$, B. Madison ${ }^{10}$, K. Mase ${ }^{17}$, T. Meures ${ }^{4}$, J. Nam ${ }^{2}$, A. Novikov ${ }^{10}$, R.J. Nichol ${ }^{6}$, G. $\mathrm{Nir}^{7}$, A. Nozdrina ${ }^{10}$, E. Oberla ${ }^{8}$, A. OMurchadha ${ }^{4}$, J. Osborn ${ }^{14}$, Y. Pan ${ }^{1}$, C. Pfendner ${ }^{5}$, N. Punsuebsay ${ }^{1}$, J. Roth ${ }^{1}$, P. Sandstrom ${ }^{4}$, D. Seckel ${ }^{1}$, Y.-S. Shiao ${ }^{2}$, A. Shultz ${ }^{10}$, D. Smith ${ }^{8}$, J. Torres ${ }^{15}$, S. Toscano ${ }^{18}$, J. Touart ${ }^{3}$, N. van Eijndhoven ${ }^{9}$, G.S. Varner ${ }^{22}$, A. Vieregg ${ }^{8}$, M.-Z. Wang ${ }^{2}$, S.-H. Wang ${ }^{2}$, Y.H. Wang ${ }^{2}$, S.A. Wissel ${ }^{11,12,20,21}$, C. Xie ${ }^{6}$, R. Young ${ }^{10}$, S. Yoshida 17 ,

${ }^{1}$ Dept. of Physics, University of Delaware, Newark, DE 19716

${ }^{2}$ Dept. of Physics, Grad. Inst. of Astrophys., Leung Center for Cosmology and Particle Astrophysics, National Taiwan University, Taipei, Taiwan

${ }^{3}$ Dept. of Physics, University of Maryland, College Park, MD 20742

${ }^{4}$ Dept. of Physics, University of Wisconsin-Madison, Madison, WI 53706

${ }^{5}$ Dept. of Physics and Astronomy, Denison University, Granville, Ohio 43023

${ }^{6}$ Dept. of Physics and Astronomy, University College London, London, United Kingdom

${ }^{7}$ Weizmann Institute of Science, Rehovot, Israel

${ }^{8}$ Dept. of Physics, Enrico Fermi Institue, Kavli Institute for Cosmological Physics, University of Chicago, Chicago, IL 60637

${ }^{9}$ Vrije Universiteit Brussel, Brussels, Belgium

${ }^{10}$ Dept. of Physics and Astronomy, University of Kansas, Lawrence, KS 66045

${ }^{11}$ Center for Multi-Messenger Astrophysics, Institute for Gravitation and the Cosmos, Pennsylvania State University, University Park, PA 16802

${ }^{12}$ Dept. of Physics, Pennsylvania State University, University Park, PA 16802

${ }^{13}$ Moscow Engineering Physics Institute, Moscow, Russia

${ }^{14}$ Dept. of Physics and Astronomy, University of Nebraska, Lincoln, Nebraska 68588

${ }^{15}$ Dept. of Physics, Center for Cosmology and AstroParticle Physics, The Ohio State University, Columbus, OH 43210

${ }^{16}$ Dept. of Physics and Astronomy, Michigan State University, East Lansing, Michigan 48824

${ }^{17}$ Dept. of Physics, Chiba University, Chiba, Japan

${ }^{18}$ Université Libre de Bruxelles, Science Faculty CP230, B-1050 Brussels, Belgium

${ }^{19}$ Dept. Physics and Astronomy, Whittier College, Whittier, CA 90602

${ }^{20}$ Dept. of Astronomy and Astrophysics, Pennsylvania State University, University Park, PA 16802

${ }^{21}$ Physics Dept., California Polytechnic State University, San Luis Obispo, CA 93407

${ }^{22}$ Dept. of Physics and Astronomy, University of Hawaii, Manoa, HI 96822

\section{Acknowledgements}

We thank the National Science Foundation Office of Polar Programs and Physics Division for their generous support through NSF OPP-902483, Grant NSF OPP-1002483, Grant NSF 1607555, Grant NSF OPP-1359535, Grant NSF OPP-1404212, and Grant NSF OPP-2013134, and Grant NSF 2019597. K. Hughes thanks the NSF for support through DGE-1746045. We would also like to acknowledge the Belgian Funds for Scientific Research (FRS-FNRS and FWO) and the FWO programme for International Research Infrastructure (IRI). 